\title{
Aspergillus Osteomyelitis of Metatarsal
}

\author{
Sheikh Tousif Reza, Kishan Prasad H.L, Jayaprakash Shetty K. and Siddharth M. Shetty \\ Pathology, K.S Hegde Medical Academy, Karnataka (India)
}

Keywords: Osteomyelitis, Aspergillus, Metatarsal, Diabetes Mellitus

\section{Dear Sir,}

Aspergillus spp. are ubiquitous in the environment, including the air, soil, and decomposing materials. ${ }^{[1]}$ Fungal infections of the bone occur usually in patients with suppressed immunological status. ${ }^{[2]}$ Aspergillus osteomyelitis is a rare infection and the involvement of metatarsals is the rarest. This entity is often neglected and data concerning risk factors, therapies and outcome are inadequate. ${ }^{[3]}$ Here we present a case report of right foot swelling which on further evaluation, diagnosis of Aspergillus osteomyelitis of metatarsal was made. An appreciation of the possibility of osteomyelitis of the metatarsal is an important element in making an early diagnosis of this potentially crippling condition.

A 42 year old male patient presented with complaints of pain and swelling of the right foot since one year. The pain was described insidious in onset, dull aching type and aggravated on bearing weight over the right foot. On palpation tenderness was felt near the navicular and $3^{\text {rd }}$ and $4^{\text {th }}$ metatarsals head of the right foot. No sinus was seen though. He was a known case of type 2 diabetes mellitus and hypertension. Antero-posterior and lateral view X-rays of the right foot revealed a lytic lesion at the base of right metatarsal. MRI of the right foot also done which reported as marrow signal intensity noted in the base and adjacent shaft of fourth metatarsal. Later on surgical exploration of the lesional area, necrotic material found at the base of $4^{\text {th }}$ metatarsal surrounded by granulation tissue. It was curetted and the material was sent for histopathological examination. Post surgical broad spectrum antifungals started with with regular follow-ups. On histopathological examination, the lesional tissue show numerous granulomas comprising clusters of epithelioid histiocytes, in the centre of which fungal colonies of Aspergillus were seen. These fungi were seen to have hyphal forms with irregular septations and acute angle branching. With the aid of PAS and GMS staining, fungal colonies could be seen properly with hyphae. Later the patient was treated with antifungal drug clotrimazole supplemented with broad spectrum antibiotic. After six months patient was examined again with no signs of swelling or pain. The wound was healed and the patient could walk with proper gait.
Aspergillus osteomyelitis of the metatarsal is a very rare entity. In literature, Aspergillus osteomyelitis was reported in vertebrae, ribs, cranium and tibia. ${ }^{[4]} \mathrm{A}$ case of chronic osteomyelitis if not properly evaluated can lead patients to undergo unnecessary antibacterial or anti-tubercular drugs treatment. This may further complicates the situation with comorbid results. These cases are generally associated with osseous tenderness, pain, sinus tracts and/or drainage. Immune status of the patient has always been a crucial factor in the etiopathogenesis of fungal osteomyelitis ${ }^{[5]}$. In this present study too, diabetes as another debilitating disease affecting the immune system could be a major contributing factor. Radiological investigation in a case of aspergillus osteomyelitis may show osteolysis, bone erosion, periosteal reaction, abscess and sequestrum. The clinical examination and radiological investigation are sufficient enough for the diagnosis of osteomyelitis, but histopathological investigation plays a crucial role for finding the etiological agent. $\mathrm{H}$ and $\mathrm{E}$ staining helps to observe morphological features of fungi along with the surrounding tissue reaction. But one should not hesitate to take the aid of special staining like PAS and GMS, especially suspecting fungal etiology. Culture is the gold standard for diagnosis of Aspergillus ${ }^{[1]}$. Treatment of Aspergillus osteomyelitis includes antifungal therapy and surgical debridement in most cases. Surgery plays a major role in debulking and eliminating infected bone. Among the antifungals, amphotericin B, itraconazole, and voriconazole are being administered in aspergillus osteomyelitis. ${ }^{[4]}$

Aspergillus osteomyelitis though rare, is a debilitating disease. Metatarsal can be included in the spectrum of site involvement besides vertebrae, ribs and cranium. The symptoms of pain and tenderness over a bony area in a patient with associated diabetes should prompt further evaluation for osteomyelitis, especially that of Aspergillus osteomyelitis. Treatment should include both surgical and medical therapy.

\section{Acknowledgements}

I would like to thank our technical staff Mr. Girish for his help in preparing and staining slides. 


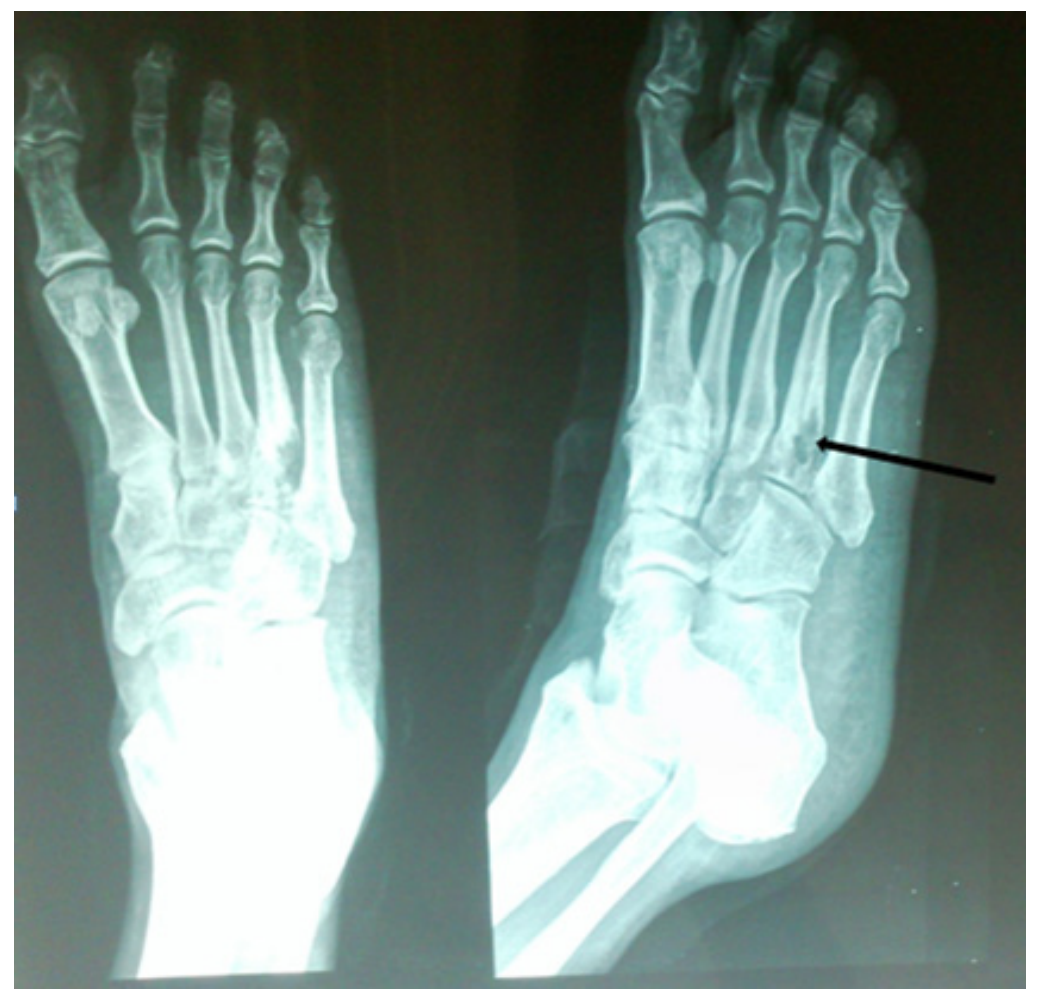

Fig. 1: Antero-posterior and lateral view Xray of right foot showing lytic lesion at the base of $4^{\text {th }}$ metatarsal.

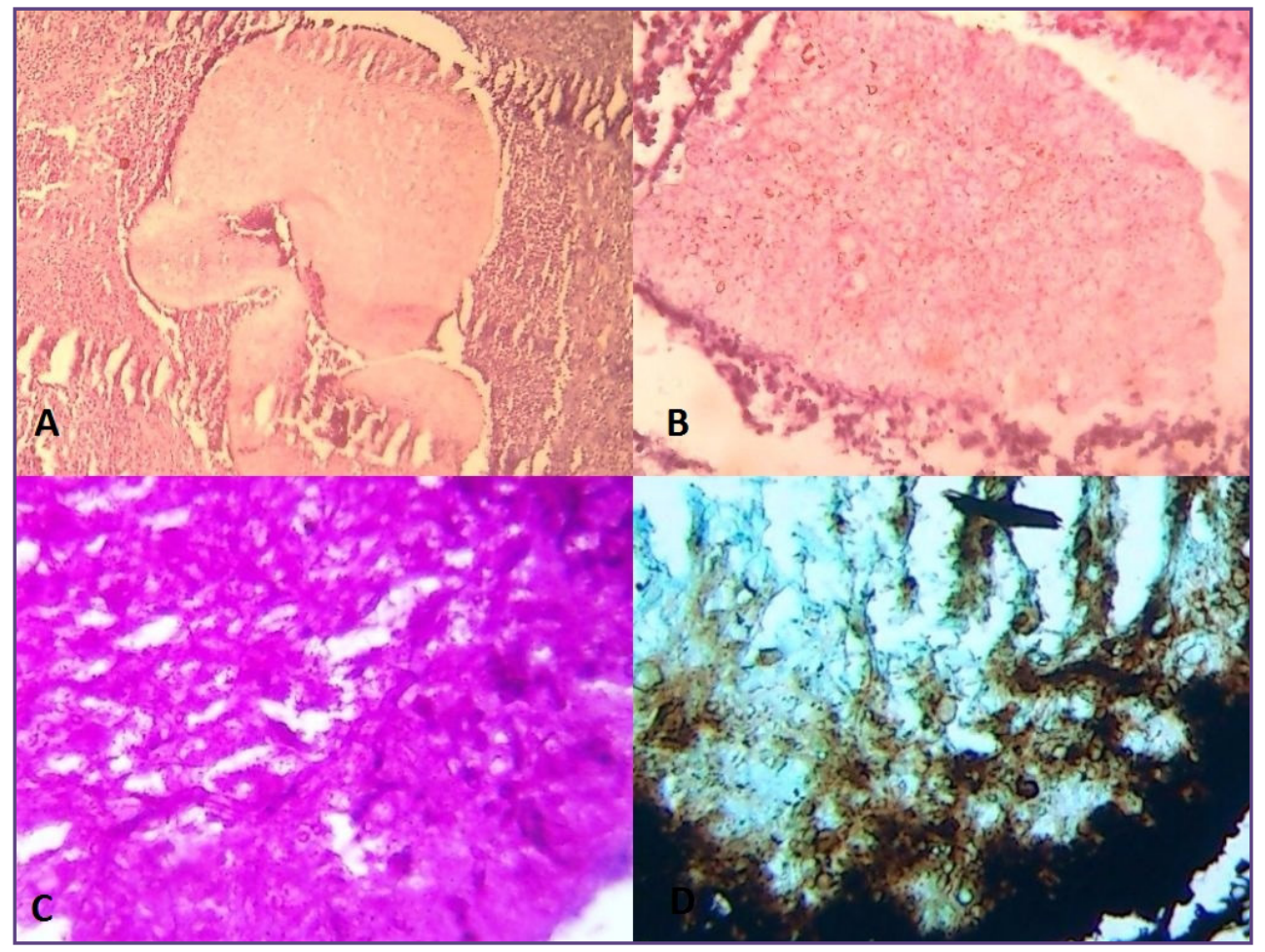

Fig. 2: [A] Fungal colonies in the centre of granuloma(H-E stain,10x) [B] Slender filamentous hyphae with irregular septation and acute angle branching (H-E stain,40x) [C] PAS stain [D] GMS stain. 


\section{Reference}

1. Horn D, Sae-Tia S, Neofytos D. Aspergillus osteomyelitis: review of 12 cases identified by the Prospective Antifungal Therapy Alliance registry. Diagnostic Microbiology and Infectious Disease. 2009;63:384-7.

2. Maheswari J. Essential Orthopaedics. $4^{\text {th }}$ edition. New Delhi: Jaypee Brothers Medical Publishers; 2011.

3. Gabrielli E, Fothergill AW, Brescini L, et al. Osteomyelitis caused by Aspergillus species: a review of 310 reported cases. Clinical Microbiology and Infection. $2014 ; 20(6)$ : 559-65.

4. Gamaletsou MN, Rammaert B, Bueno MA, et al. Aspergillus osteomyelitis: epidemiology, clinical manifestations, management, and outcome. Journal of Infection. 2014 ;68(5):478-93.

5. Sethi S, Siraj F, Kalra KL, Chopra P. Aspergillus vertebral osteomyelitis in immunocompetent patients. Indian journal of orthopaedics. 2012;46(2):246.

*Corresponding author:

Dr. Kishan Prasad H.L, K S Hegde Medical Academy, P.O Deralakatte, Mangaluru (India) - 575018

Phone: +91 7411941096

Email: dr_kishanprasad@yahoo.in

Financial or other Competing Interests: None. 